\title{
Depressão em idosos institucionalizados
}

\author{
Depression in institutionalized elderly \\ Depresión en ancianos institucionalizados
}

Ariella Sthefany Silva Oliveira ORCID: https://orcid.org/0000-0002-0123-3119 Centro Universitário Unifacid Wyden, Brasil E-mail: popyariella@gmail.com Alice Lima Rosa Mendes ORCID: https://orcid.org/0000-0002-1960-9647 Universidade de Brasília, Brasil E-mail: alice_lima_hotmail.com

Sara Ferreira Lobato de Brito ORCID: https://orcid.org/0000-0001-8207-7906 Centro Universitário Unifacid Wyden, Brasil E-mail: Saraflbrito99@gmail.com

Rodrigo Feitosa de Oliveira Correia ORCID: https://orcid.org/0000-0002-8614-5555 Centro Universitário Unifacid Wyden, Brasil E-mail: rodrigofeitosatsb@gamail.com

Luís Paulo Alves Ramos

ORCID: https://orcid.org/0000-0002-0196-8860 Universidade Federal de São Carlos, Brasil E-mail: to.luispauloramos@gmail.com Bruna Correa Nolêto

ORCID: https://orcid.org/0000-0001-7371-1936 Centro Universitário Unifacid Wyden, Brasil

E-mail: brunacnoleto@gmail.com

Samyres Batista De Medeiros

ORCID: https://orcid.org/0000-0001-5647-5783 Centro Universitário Uninovafapi, Brasil

E-mail: samyresmed28@gmail.com

Ivanildes do Nascimento

ORCID: https://orcid.org/0000-0002-9988-8817

Centro Universitário Unifacid Wyden, Brasil

E-mail: ivanildesgda@hotmail.com

Ivani Feitosa de Oliveira

ORCID: https://orcid.org/0000-0002-0662-8785 Universidade Federal do Piauí, Brasil E-mail: ivannioliveira@hotmail.com

Andreza Lima Cavalcante

ORCID: https://orcid.org/0000-0002-1965-4405 Centro Universitário Unifacid Wyden, Brasil

E-mail: andreza.lima.cavalcante@gmail.com Suyane Santana Cavalcante ORCID: https://orcid.org/0000-0002-1675-8380 Centro Universitário Unifacid Wyden, Brasil E-mail: cavalcantesuy99@gmail.com

Fabíola de Oliveira Alvino Macêdo ORCID: https://orcid.org/0000-0003-1023-4399 Universidade do Estado do Pará, Brasil E-mail: fabiolaalvino@gmail.com

Gabriela Dantas Carvalho

ORCID: https://orcid.org/0000-0002-9571-3323 Universidade Federal do Delta do Parnaíba, Brasil

E-mail: ftgabrieladantas@hotmail.com

Silvana Maria Véras Neves

ORCID: https://orcid.org/0000-0003-4784-9135

Universidade de Fortaleza, Brasil

E-mail: silvanafisio7@yahoo.com.br 


\title{
Resumo
}

Com a transição demográfica ocorrida no Brasil e no mundo, houve uma redução na mortalidade e aumento da longevidade, evidenciando-se uma população composta por pessoas da terceira idade. A depressão acomete uma boa parcela dos idosos, principalmente os que vivem em instituições de longa permanência (ILP), distantes de suas famílias. O objetivo geral desse estudo foi investigar os principais fatores associados à depressão em idosos institucionalizados. Tratou- se de uma revisão integrativa. A estratégia de busca foi realizada nas bases de dados informatizadas de acesso gratuito para pesquisa de artigos científicos, disponibilizadas pela Biblioteca Virtual em Saúde (BVS), Google Acadêmico (GA), e no Portal de Periódicos CAPES, no período de janeiro a abril de 2020, utilizando-se os seguintes descritores: "depression". "elderly" e "institutionalization" com o operador booleano "AND" para a especificidade do levantamento bibliográfico. Os critérios de inclusão definidos para a seleção foram: artigos publicados na língua portuguesa que retratassem a temática, publicados e indexados nos referidos bancos de dados nos últimos cinco anos, sendo excluídos aqueles que não estavam disponíveis na íntegra. Foram obtidos 234 artigos, mas pelos critérios de inclusão e exclusão estabelecidos, 10 estudos foram selecionados para compor a amostra final. Verificou-se que a depressão nos idosos institucionalizados é uma realidade, bem como a presença de várias comorbidades, incapacidade funcional, pouco engajamento nas atividades e pouca participação social, são fatores que influenciam na depressão e, portanto, na qualidade de vida desses sujeitos. Concluiu-se que o tempo de institucionalização, a carência das relações interpessoais e a solidão constituíram fatores de risco para a depressão.

Palavras-chave: Depressão; Idoso; Institucionalização.

\begin{abstract}
With the demographic transition that took place in Brazil and in the world, there was a reduction in mortality and an increase in longevity, evidencing a population composed of elderly people. Depression affects a good portion of the elderly, especially those living in long-term care facilities (ILP), far from their families. The general objective of this study was to investigate the main factors associated with depression in institutionalized elderly people. It was an integrative review. The search strategy was carried out in computerized databases with free access to search for scientific articles, made available by the Virtual Health Library (BVS), Academic Google (GA), and the CAPES Journal Portal, from January to April 2020, using the following descriptors: "depression". "elderly" and "institutionalization" with the Boolean operator "AND" for the specificity of the bibliographic survey. The inclusion criteria defined for the selection were: articles published in Portuguese that portrayed the theme, published and indexed in the aforementioned databases in the last five years, excluding those that were not available in full. A total of 234 articles were obtained, but according to the established inclusion and exclusion criteria, 10 studies were selected to make up the final sample. It was found that depression in institutionalized elderly is a reality, as well as the presence of various comorbidities, functional disability, little engagement in activities and little social participation, are factors that influence depression and, therefore, the quality of life of these subjects. It was concluded that the length of institutionalization, the lack of interpersonal relationships and loneliness constituted risk factors for depression.
\end{abstract}

Keywords: Depression; Elderly; Institutionalization.

\section{Resumen}

Con la transición demográfica que se produjo en Brasil y en el mundo, hubo una reducción de la mortalidad y un aumento de la longevidad, evidenciando una población compuesta por personas mayores. La depresión afecta a una buena parte de los ancianos, especialmente a los que viven en centros de atención a largo plazo (ILP), lejos de sus familias. El objetivo general de este estudio fue investigar los principales factores asociados a la depresión en ancianos institucionalizados. Fue una revisión integradora. La estrategia de búsqueda se realizó en bases de datos computarizadas de libre acceso para la búsqueda de artículos científicos, puestas a disposición por la Biblioteca Virtual en Salud (BVS), Google Académico (GA) y el Portal de Revistas CAPES, de enero a abril de 2020, utilizando los siguientes descriptores: "Depresión". "Anciano" e "institucionalización" con el operador booleano "Y" para la especificidad de la encuesta bibliográfica. Los criterios de inclusión definidos para la selección fueron: artículos publicados en portugués que retrataran el tema, publicados e indexados en las bases de datos mencionadas en los últimos cinco años, excluyendo aquellos que no estaban disponibles en su totalidad. Se obtuvieron un total de 234 artículos, pero de acuerdo con los criterios de inclusión y exclusión establecidos, se seleccionaron 10 estudios para conformar la muestra final. Se encontró que la depresión en ancianos institucionalizados es una realidad, así como la presencia de diversas comorbilidades, discapacidad funcional, poca participación en actividades y poca participación social, son factores que influyen en la depresión y, por ende, en la calidad de vida de estos sujetos. Se concluyó que el momento de la institucionalización, la falta de relaciones interpersonales y la soledad constituían factores de riesgo para la depresión.

Palabras clave: Depresión; Anciano; Institucionalización. 


\section{Introdução}

Este estudo aborda a depressão em idosos institucionalizados, entendendo que além dessa matéria abranger um importante indicador do quadro de saúde da população na terceira idade, possibilita refletir sobre a qualidade da atenção direcionada às pessoas idosas residentes nas Instituições de Longa Permanência para Idosos (ILPI), com foco nas suas particularidades e nas ações necessárias para um envelhecimento saudável e com qualidade de vida (Veras \& Oliveira, 2018).

As projeções da Organização Mundial da Saúde (OMS), de que até 2050 se terá um contingente populacional crescente de idosos no país, as doenças que surgem em meio às características peculiares do processo de envelhecimento, como a depressão por exemplo, requerem uma melhor compreensão sobre os fatores que a constituem, tendo em vista que vem ganhando maior expressão na sociedade, por representar um grave problema de saúde pública, constituindo preocupação de especialistas do campo da saúde geriátrica e afins (Belasco \& Okuno, 2019).

De acordo com estimativas elaboradas pela OMS, e divulgadas em 2018 pelo Instituto Brasileiro de Geografia e Estatística (IBGE), o número de idosos no Brasil deverá aumentar. Por volta do ano de 2060, a população idosa brasileira poderá atingir cerca de 173,47\% (Belasco \& Okuno, 2019).

Cabe ressaltar que, frente a essas estimativas, surge a necessidade de se buscar alternativas para manter as pessoas idosas socialmente e economicamente integradas e independentes. Contudo, ressalta- se que a idade e o processo de envelhecimento possuem suas peculiaridades e dentre essas, citam-se a vulnerabilidade e redução da capacidade funcional dessas pessoas nesse momento da vida, além de outros aspectos capazes de acarretar sentimentos que contribuem para o quadro sintomático da depressão (Lampert \& Ferreira, 2018).

No que diz respeito à condição de moradia e lugar de convivência desse idoso em uma ILPI, leva a criação de novas possibilidades de assistência que lhe garanta a manutenção de melhor qualidade de vida, em todos os aspectos a fim de se fazer valer essa premissa, a busca por fatores, que contribuem para o surgimento da depressão em idosos que se encontram institucionalizados (Miranda, Mendes \& Silva, 2016).

A institucionalização do idoso tem sido associada as dificuldades econômicas e psicossociais das famílias para o cuidado, sobretudo pela vulnerabilidade e redução da capacidade funcional das pessoas nesse momento da vida. Os profissionais das ILPIs devem estar capacitados para lidar diretamente com a situação do idoso, preservando a sua qualidade de vida. As instituições deveriam fornecer condições favoráveis em todos os aspectos para o idoso, porém, por dificuldades financeiras, isso nem sempre se confirma. Daí, o interesse pelo tema, que surgiu a partir das leituras abrangendo as necessidades de idosos que residem em uma ILP, convivendo com outros idosos em isolamento, longe dos seus familiares (Pinheiro et al., 2016),(Fagundes et al, 2017).

Partindo dessa perspectiva e considerando a importância das experiências a serem aferidas para a compreensão da realidade nacional quanto ao quadro de depressão na população idosa brasileira, esta pesquisa teve como questionamento: quais evidências constam nas publicações selecionadas, referentes aos fatores associados à depressão em idosos institucionalizados? Para tanto, o objetivo consistiu em investigar os principais fatores associados à depressão em idosos institucionalizados, a partir da identificação desses agentes, nas publicações científicas nacionais de 2016 à 2020.

\section{Metodologia}

Trata-se de uma pesquisa bibliográfica, por meio de uma revisão integrativa da literatura, acerca da depressão em idosos institucionalizados. A revisão integrativa pode proporcionar a síntese de conhecimento e a inclusão da aplicação de resultados e estudos significativos na prática. A estratégia de busca, foi realizada nas bases de dados informatizadas de acesso gratuito para pesquisa de artigos científicos, disponibilizadas pela Biblioteca Virtual em Saúde (BVS), Google Acadêmico (GA), e no Portal 
de Periódicos CAPES, no período de janeiro a abril de 2021, utilizando-se os seguintes descritores: "depression". "elderly" e "institutionalization" com o operador booleano "AND" para a especificidade do levantamento bibliográfico.

O período considerado, para a revisão foi o de 2016 a 2020. Os critérios de inclusão e exclusão de artigos foram selecionados a partir do título e da leitura dos resumos segundo os seguintes critérios de inclusão: deveriam referir-se à depressão em idosos institucionalizados, publicados na língua portuguesa e indexados nos referidos bancos de dados nos últimos cinco anos. Os que não obedeceram a esses critérios e nem estavam disponíveis na íntegra, bem como os artigos de revisão de literatura, dissertações e teses foram excluídos.

Na BVS foram encontrados 89 artigos, excluídos 85 , sendo selecionados 4 trabalhos, enquanto no GA foram encontrados 102 artigos, descartados 97 e selecionados 5, e no portal de periódicos CAPES, foram encontrados 43 artigos, excluídos 42 e selecionado 1 artigo. No total foram selecionados 10 trabalhos entre 2016 e 2020.

Diante disso, localizou-se 234 trabalhos, dos quais 224 foram excluídos 24 por se repetirem em mais de uma base de dados, 90 artigos por se tratarem de revisão sistemática e/ou revisões narrativas, 32 por serem livros, 12 por inconsistência nas informações e 66 por não tratarem do tema da pesquisa. Para a análise da revisão integrativa permaneceram 10 artigos. A organização e análise dos dados na primeira etapa foi elaborada a questão norteadora; na segunda etapa foram estabelecidos os parâmetros de inclusão e exclusão; na terceira etapa foi realizada a coleta nas bases de dados científicas; na quarta etapa foi feita a seleção e análise dos artigos; na quinta etapa realizou-se a interpretação dos resultados e, finalmente, os resultados da revisão.

\section{Resultados e Discussão}

As dez publicações selecionadas foram distribuídas em uma tabela de acordo com as variáveis: autor/ano, título, objetivo geral, tipo de estudo e conclusão (Tabela 1). Após esse procedimento, foi realizada uma análise descritiva dos resultados evidenciados e efetuada a discussão, de acordo com a literatura pertinente referenciada.

A seguir encontram-se dispostos os 10 artigos utilizados nesta revisão integrativa, com os resultados da pesquisa realizada, de acordo com as variáveis do estudo.

Tabela 1. Artigos selecionados para a pesquisa.

\begin{tabular}{|c|c|c|c|c|c|}
\hline $\mathbf{N}^{\mathbf{o}}$ & $\begin{array}{l}\text { Autor } \\
\text { Ano }\end{array}$ & Título & Objetivo Geral & Tipo de Estudo & Conclusão do Estudo \\
\hline 1 & 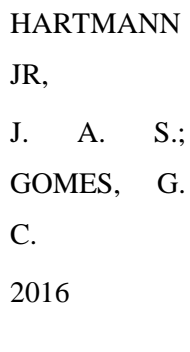 & $\begin{array}{l}\text { Depressão em idosos } \\
\text { institucionaliza-dos: } \\
\text { padrões cognitivos e } \\
\text { qualidade de vida }\end{array}$ & $\begin{array}{lr}\text { Determinar } & \text { o } \\
\text { percentual de } & \\
\text { depressão,variáveis } & \\
\text { psicossociais } & \text { e } \\
\text { qualidade de vida de } \\
\text { idosos } \\
\text { institucionalizados }\end{array}$ & $\begin{array}{l}\text { Estudo descritivo, } \\
\text { transversal, } \\
\text { observacio-nal, com } \\
\begin{array}{l}\text { comparação } \\
\text { grupos. }\end{array}\end{array}$ & $\begin{array}{l}\text { A identificação do alto percentual de } \\
\text { depressão ( } 63 \% \text { de } 42 \text { idosos) demanda } \\
\text { ressaltar o novo paradigma da saúde pública, } \\
\text { no que concerne à população de idosos. }\end{array}$ \\
\hline 2 & $\begin{array}{l}\text { MORAES B. } \\
\text { S de; et al. } \\
2016\end{array}$ & $\begin{array}{l}\text { Sintomas da depressão } \\
\text { associada ao abandono } \\
\text { em idosos } \\
\text { institucionaliza-dos } \\
\text { nos municípios de } \\
\text { Firminópolis e São } \\
\text { Luís De Montes } \\
\text { Belos-Goiás }\end{array}$ & $\begin{array}{lr}\text { Analisar os } & \text { sintomas } \\
\text { específicos } & \text { da } \\
\text { depressão associado ao } \\
\text { abandono } \\
\text { munícipios }\end{array}$ & $\begin{array}{l}\text { Estudo descritivo de } \\
\text { caráter quantitativo }\end{array}$ & $\begin{array}{l}\text { O resultado de } 80 \% \text { dos } 21 \text { idosos } \\
\text { entrevistados apresentarem algum sintoma } \\
\text { depressivo apontam a grande necessidade de } \\
\text { acompanhamento de saúde mental em } \\
\text { instituições de longa permanência (ILPI). }\end{array}$ \\
\hline
\end{tabular}




\begin{tabular}{|c|c|c|c|c|c|}
\hline & & & $\begin{array}{l}\text { das instituições } \\
\text { públicas Lar Bom } \\
\text { Samaritano e na Casa } \\
\text { do Idoso Geraldo } \\
\text { Francisco Chavier. }\end{array}$ & & \\
\hline 3 & $\begin{array}{l}\text { NÓBREGA I, } \\
\text { P.; LEAL, M. } \\
\text { C. C.; } \\
\text { MARQUES, } \\
\text { A. P. de O. } \\
2016 .\end{array}$ & $\begin{array}{l}\text { Prevalência de } \\
\text { sintomas depressivos e } \\
\text { fatores associados em } \\
\text { idosos } \\
\text { institucionaliza-dos no } \\
\text { município de Recife, } \\
\text { Pernambuco }\end{array}$ & $\begin{array}{l}\text { Investigar a prevalência } \\
\text { de sintomas depressivos } \\
\text { e seus possíveis fatores } \\
\text { associados em idosos } \\
\text { institucionaliza-dos no } \\
\text { município de Recife, } \\
\text { Pernambuco. }\end{array}$ & $\begin{array}{l}\text { Estudo descritivo, } \\
\text { transversal e } \\
\text { quantitativo. }\end{array}$ & $\begin{array}{l}\text { A alta prevalência de sintomas depressivos } \\
\text { na população institucionalizada alerta para a } \\
\text { necessidade de maior engajamento dos } \\
\text { gestores e profissionais da saúde, na } \\
\text { prevenção, investigação e na valorização dos } \\
\text { sinais indicativos de depressão }\end{array}$ \\
\hline 4 & $\begin{array}{l}\text { RALDI, G. V; } \\
\text { CANTELE, } \\
\text { A. B; } \\
\text { PALMEIRAS, } \\
\text { G. de B. } \\
2016\end{array}$ & $\begin{array}{l}\text { Avaliação da } \\
\text { prevalência de } \\
\text { depressão em idosos } \\
\text { institucionaliza-dos } \\
\text { em uma ILPI no norte } \\
\text { do RS. }\end{array}$ & $\begin{array}{l}\text { Detectar casos } \\
\text { prevalentes de idosos } \\
\text { com depressão e a } \\
\text { suspeita de novos casos, } \\
\text { após a aplicação da } \\
\text { Escala de Depressão } \\
\text { Geriátrica de Yesavage. }\end{array}$ & $\begin{array}{l}\text { Estudo de caráter } \\
\text { exploratório-analítico } \\
\text { e quantitativo, }\end{array}$ & $\begin{array}{l}\text { A maioria da população alvo apresentou um } \\
\text { quadro de normalidade após a aplicação da } \\
\text { Escala de Depressão Geriátrica de Yesavage, } \\
\text { o que traz um resultado positivo para o } \\
\text { estudo, mas que não desmerece a presença } \\
\text { dos casos de depressão encontrados, } \\
\text { salientando que os mesmos podem estar } \\
\text { relacionados a inúmeras questões, não sendo } \\
\text { a institucionalização o principal. }\end{array}$ \\
\hline 5 & $\begin{array}{l}\text { SILVA, C. S; } \\
\text { et al. } \\
2016\end{array}$ & $\begin{array}{l}\text { A prevalência e alguns } \\
\text { fatores de depressão } \\
\text { em idosos } \\
\text { institucionaliza-dos }\end{array}$ & $\begin{array}{l}\text { Investigar a prevalência } \\
\text { e fatores de depressão } \\
\text { em idosos residentes } \\
\text { em Instituição de Longa } \\
\text { Permanência (ILPIs). }\end{array}$ & $\begin{array}{l}\text { Pesquisa } \\
\text { exploratória, } \\
\text { bibliográfica e de } \\
\text { campo, com caráter } \\
\text { qualitativo } \\
\text { quantitativo. }\end{array}$ & $\begin{array}{l}\text { Necessidade de atenção maior direcionada ao } \\
\text { idoso favorecendo assim melhoria na } \\
\text { qualidade de vida desses. Uma das formas de } \\
\text { ajudar o idoso a resgatar sua autonomia pode } \\
\text { ser a prática de exercício físico, } \\
\text { proporcionando uma melhora psicossocial, } \\
\text { diminuindo a sintomatologia depressiva, } \\
\text { além de reduzir o efeito do envelhecimento e } \\
\text { prevenir outras doenças }\end{array}$ \\
\hline 6 & $\begin{array}{l}\text { KRATZ, V. C. } \\
\text { L; et al. } \\
2018\end{array}$ & $\begin{array}{l}\text { Promoção de saúde de } \\
\text { idosos } \\
\text { institucionaliza-dos e } \\
\text { crenças quanto ao } \\
\text { envelhecer: projeto } \\
\text { intergeracional. }\end{array}$ & $\begin{array}{l}\text { Verificar se uma } \\
\text { intervenção } \\
\text { intergeracional de troca } \\
\text { de cartas influenciaria } \\
\text { mudanças nos níveis de } \\
\text { depressão em idosos } \\
\text { institucionaliza-dos, e } \\
\text { modificaria crenças } \\
\text { sobre a velhice em } \\
\text { jovens } \\
\text { universitário adultos }\end{array}$ & $\begin{array}{l}\text { Pesquisa empírica, } \\
\text { com método misto, } \\
\text { delineamento } \\
\text { quantitativo } \\
\text { longitudinal- } \\
\text { prospectivo e } \\
\text { qualitativo analítico. }\end{array}$ & $\begin{array}{l}\text { Os resultados indicaram que a intervenção } \\
\text { teve potencial capacidade de influenciar a } \\
\text { modificação de crenças quanto ao } \\
\text { envelhecer, e de reduzir os níveis de } \\
\text { depressão em } 5 \text { dos } 7 \text { idosos participantes da } \\
\text { pesquisa, e moradores distribuídos em duas } \\
\text { ILPIs, localizadas no interior do Rio Grande } \\
\text { do Sul. }\end{array}$ \\
\hline 7 & $\begin{array}{l}\text { MELO L. A; } \\
\text { et al. } \\
2018\end{array}$ & $\begin{array}{l}\text { Fragilidade, } \\
\text { sintomas depressivos e } \\
\text { qualidade de vida: um } \\
\text { estudo com idosos }\end{array}$ & $\begin{array}{l}\text { Analisar a relação entre } \\
\text { fragilidade, sintomas } \\
\text { depressivos e qualidade } \\
\text { de vida de idosos }\end{array}$ & $\begin{array}{l}\text { Estudo descritivo, } \\
\text { correlacional, de } \\
\text { corte transversal, }\end{array}$ & $\begin{array}{l}\text { Idosos institucionalizados frágeis e com } \\
\text { sintomas depressivos apresentaram pior } \\
\text { percepção de qualidade de vida. }\end{array}$ \\
\hline
\end{tabular}


Research, Society and Development, v. 10, n. 10, e130101018620, 2021

(CC BY 4.0) | ISSN 2525-3409 | DOI: http://dx.doi.org/10.33448/rsd-v10i10.18620

\begin{tabular}{|c|c|c|c|c|c|}
\hline & & institucionaliza-dos. & institucionalzados. & & \\
\hline 8 & $\begin{array}{l}\text { FRUTUOSO, } \\
\text { E. A; et al. } \\
2019\end{array}$ & $\begin{array}{l}\text { Idosos } \\
\text { institucionaliza-dos e } \\
\text { depressão: } \\
\text { rastreamento dos } \\
\text { sintomas }\end{array}$ & $\begin{array}{l}\text { Analisar a prevalência } \\
\text { de sintomas depressivos } \\
\text { em idosos } \\
\text { institucionaliza-dos }\end{array}$ & $\begin{array}{l}\text { Estudo descritivo, } \\
\text { com abordagem } \\
\text { quantitativa, }\end{array}$ & $\begin{array}{l}\text { Torna-se primordial a criação de programas } \\
\text { para idosos institucionalizados que visem } \\
\text { promover participações diretas no âmbito } \\
\text { social, cultural, esportivo, lazer e } \\
\text { educacional, com ênfase no empoderamento } \\
\text { dos atores sociais e na redução da } \\
\text { sintomatologia depressiva neste grupo etário. }\end{array}$ \\
\hline 9 & $\begin{array}{l}\text { GUIMARÃES } \\
\text {, L. de A; et al. } \\
2019\end{array}$ & $\begin{array}{l}\text { Sintomas depressivos } \\
\text { e fatores associados } \\
\text { em idosos residentes } \\
\text { em instituição de } \\
\text { longa permanência. }\end{array}$ & $\begin{array}{l}\text { Verificar a prevalência } \\
\text { e fatores associados a } \\
\text { sintomas depressivos } \\
\text { em idosos } \\
\text { institucionaliza-dos. }\end{array}$ & $\begin{array}{l}\text { Estudo epidemiológi- } \\
\cos \quad \text { com } \\
\text { delineamento } \\
\text { transversal }\end{array}$ & $\begin{array}{l}\text { Alta prevalência de sintomas depressivos em } \\
\text { idosos institucionalizados, associado às } \\
\text { variáveis presença de incontinência urinária, } \\
\text { auto percepção de saúde (negativa), } \\
\text { qualidade de sono (ruim) e aposentadoria } \\
\text { (sim). Através do estudo e diante das } \\
\text { necessidades enfrentadas por essa população, } \\
\text { faz-se necessário a busca por medidas que } \\
\text { atuem diretamente nas variáveis } \\
\text { modificáveis, prevenindo e tratando-as. }\end{array}$ \\
\hline 10 & $\begin{array}{l}\text { MAIA, R. P; } \\
\text { et al. } \\
2020\end{array}$ & $\begin{array}{l}\text { Depressão em idosos } \\
\text { institucionaliza-dos }\end{array}$ & $\begin{array}{l}\text { Verificar a presença da } \\
\text { depressão em idosos } \\
\text { institucionaliza-dos na } \\
\text { região da Mata Norte do } \\
\text { estado de Pernambuco }\end{array}$ & $\begin{array}{l}\text { Pesquisa de } \\
\text { quantitativa, compo } \\
\text { coleta de dados } \\
\text { indireta }\end{array}$ & $\begin{array}{l}\text { A depressão se mostrou, entre os idosos } \\
\text { institucionaliza-dos, mais prevalente em } \\
\text { homens do que em mulheres, e o } \\
\text { acompanhamento psicológico ou } \\
\text { psiquiátrico mostrou-se precário }\end{array}$ \\
\hline
\end{tabular}

Fonte: Autores (2021).

De acordo com a literatura o contexto institucional favorece a vivência de perdas em diversos aspectos, aumento da vulnerabilidade e quadros depressivos, que podem agravar patologias pré-existentes, desencadear outras desordens psiquiátricas e perda da autonomia (Gullich, Duro \& Cesar, 2016).

Conforme (Lopes et al. 2018) e (Lampert \& Ferreira 2018) os idosos institucionalizados vivenciam situações que podem aumentar sua vulnerabilidade aos transtornos depressivos, tais como a mudança brusca e repentina no estilo de vida, o confinamento, o isolamento e a separação do seio familiar. A estimativa é de que com o processo de envelhecimento, as pessoas perdem gradativamente a sua dinâmica funcional, assim como tem autonomia reduzida, o que pode ser sentido como condição que proporciona abandono, exclusão, fazendo com que esses idosos apresentem sintomas depressivos, mais acentuadamente, as populações institucionalizadas.

Segundo, Hartmann Jr. \& Gomes (2016) investigaram algumas características sociodemográficas de 95 idosos (idade mediana de 78 anos) em nove ILPI, e, além da avaliação da qualidade de vida do grupo, identificaram depressão em 63,5\% do total de idosos avaliados pela Escala de Depressão Geriátrica (EDG), e em 59,4\%, no MiniMeem 5.0 (Mini exame do Estado Mental). Evidenciaram idosos com depressão grave, com depressão leve, e pontuação incompatível com depressão e também que apontou que o duo "depressão e perdas cognitivas" se associaram à menor condição de letramento, ausência ou raridade de visitas familiares, de saída da instituição, de submissão aos exames de rotina e à maior complexidade da ocupação anterior à institucionalização. 
De acordo com Matias et al. (2016) e (Martins \& Guimarães, 2017), também corroboram que o contexto institucional favorece ao idoso vivenciar perdas em vários aspectos da vida, contribuindo para o aumento da vulnerabilidade e também aos quadros depressivos, capazes de desencadear alterações de ordem psíquica, além de restrição da autonomia e agravamento de patologias preexistentes. A dependência funcional, assim como a deterioração do apoio familiar e o distanciamento dos familiares, podem ser fatores potencializadores dos sintomas da depressão, levando o idoso a se sentir solitário e na situação de isolamento afetivo, alimentando sentimentos de tristeza e de abandono.

Os estudos de (Moraes et al. 2016), (Silva et al. 2016) e (Kratz et al. 2018), trazem dados referentes a motivos variados, para a presença das sintomatologias depressivas, como o sentimento de abandono, por exemplo, no estudo descritivo de (Moraes et al. 2016) a análise que abrangeu a associação dos sintomas específicos da depressão e abandono em 21 idosos residentes em duas ILPIs (Lar Bom Samaritano e na Casa do Idoso Geraldo Francisco Chavier), nos munícipios de Firminópolis e São Luís dos Montes Belos respectivamente, evidenciou que dentre $80 \%$ do total do grupo entrevistado $17 \%$ eram idosas e $83 \%$ idosos, com idade entre 60 e 69 anos (8\% e 33\%); entre 70 a 79 anos (9\% e $48 \%$ ); entre 80 a 89 anos (4\% e 19\%), apresentaram algum sintoma depressivo.

Em conformidade com o mencionado por (Lopes et al., 2018) e (Lampert \& Ferreira 2018), os resultados de (Moraes et al. 2016) identificaram, dentre as sintomatologias referidas, o sentimento de solidão, mesmo convivendo junto com outros idosos na ILPI, a perda de familiares, abandono, incapacidade, aborrecimento frequente, tristeza, gostar de se isolar, não ter vontade de conversar e dificuldades para dormir. Seis idosos da Casa do Idoso Geraldo Francisco Chavier estavam fazendo acompanhamento no Centro de Atenção Psicossocial (CAPS), por conta do diagnóstico depressivo (predominando pessoas idosas do sexo masculino, 75\%). O sentimento de abandono, dos idosos das duas ILPIs prevaleceu, sugerindo que foi uma das principais causas da depressão apresentada pelos idosos.

O estudo de (Nóbrega, Leal \& Marques 2016), desenvolvido em nove Instituições de Longa Permanência de Idosos (ILPIs) em Recife, para investigar a prevalência de sintomas depressivos e seus possíveis fatores associados em 136 idosos institucionalizados, com idades entre 60 e 104 anos, apontou, por meio da Escala de Depressão Geriátrica de 15 itens (EDG15), que a prevalência de quadro depressivo foi de $53,7 \%$, com sintomatologia do tipo leve $(44,1 \%)$ ou severa $(9,6 \%)$.

A pesquisa de (Nóbrega, Leal \& Marques 2016), identificou o sexo, o estado civil, a saúde autopercebida e a funcionalidade como variáveis que se associaram ao risco de depressão dos idosos institucionalizados, visto que, os sintomas de depressão prevaleceram nos idosos do sexo feminino, nos separados ou divorciados, nos que apresentavam algum tipo de restrição, nos que tinham percepção ruim da própria saúde e nos que apresentavam dependência funcional para Atividades de Vida Diária (AVD).

De acordo com o estudo desenvolvido por (Raldi, Cantele \& Palmeiras 2016), é possível que o idoso com quadro de depressão geriátrica vivencie baixa qualidade de vida, além da degradação no seu estado de saúde geral. Daí, a importância dos profissionais da saúde e familiares, minimizarem as consequências da institucionalização, atentando para os aspectos que contribuem para aos agravos à saúde mental dos idosos.

Segundo Raldi, Cantele \& Palmeiras (2016) realizaram a pesquisa em uma ILPI, no município de Erechim - Rio Grande do Sul, abrangendo 60 idosos com idade entre 60 a 100 anos, para detectar casos prevalentes de depressão e a suspeita de novos casos, após a aplicação da Escala de Depressão Geriátrica de Yesavage, sendo revelado que, mesmo com a evidência dos casos de depressão leve (23/38\%) e de depressão grave (3/05\%), a maioria da população idosa residente na ILPI também apresentou um quadro de normalidade (34/57\%). O resultado apontou que os casos de depressão podiam estar relacionados à inúmeros elementos, além do referente ao ambiente social da institucionalização.

Na pesquisa de Silva et al. (2016) com 8 idosos, na faixa de idade entre 60 e 80 anos acima, de uma população de 15 residentes em uma instituição de acolhimento situada na cidade de Goiânia - GO, para investigar a prevalência e fatores de 
depressão, evidenciou-se que 77\% dos idosos apresentaram depressão grave e 33\% moderada, cujos fatores depressivos predominantes identificados, foram: prejuízo funcional, social e cognitivo (100\%); diminuição da qualidade de vida (100\%); perda de autonomia (75\%); sentimento de solidão (75\%); sofrimento com a ausência familiar (75\%) e falta de lazer (75\%), confirmando nesses resultados que o surgimento da depressão se deu por diversos fatores, como apontado no estudo de (Gullich, Duro \& Cesar 2016) e de (Lampert \& Ferreira 2018).

De acordo com, Kratz et al., (2018) verificaram se uma intervenção intergeracional de troca de cartas influenciaria mudanças nos níveis de depressão em idosos institucionalizados, revelando que do total de 07 idosos participantes da pesquisa, distribuídos em duas ILPIs, localizadas no interior do Rio Grande do Sul, 05 idosos apresentavam sintomas depressivos antes da intervenção (4 idosas - 1 com depressão severa, 3 com depressão leve; e 1 idoso - com depressão leve). Os dois idosos restantes do grupo ( 1 idosa e 1 idoso), apresentaram suspeita de depressão. Após a intervenção, três ( 2 idosas e 1 idoso), melhoraram seu estado emocional.

Ainda sobre o estudo de Kratz et al., (2018), os relatos de quatros idosos do grupo referiram expressões envolvendo depressão, solidão e/ou desesperança: "o meu maior problema é não ter com quem comentar as minhas preferências, não tenho com quem conversar" (Idosa, 89 anos, 01 ano na ILPI); "estou triste, pois minha vida não foi como eu quis" (Idosa, 65 anos, 11 meses na ILPI); "meu filho reformou toda minha casa e não pude aproveitar nada, tive que vir para cá" (Idosa, 78 anos, 03 meses na ILPI); “desde que perdi meus filhos não durmo sem calmante” (Idosa, 86 anos, 04 meses na ILPI).

Essas averbações nas correspondências confirmaram que o estado depressivo desses idosos, detectado pela Escala de Depressão Geriátrica (GDS) e referido na literatura pertinente, está associado ao momento avançado de vida e às perdas envolvidas, iminência da finitude e possível agravamento pela institucionalização. Tal como exposto nos estudos de (Matias et al. 2016) e de (Lima et al. 2016), a institucionalização, em alguns casos, traz ao idoso a dificuldade de adaptação, o que impacta de maneira negativa na sua qualidade de vida, além de abalar a sua independência e autonomia.

Segundo apontamentos teóricos em (Kratz et al. 2018) a qualidade de vida dos idosos institucionalizados depende de fatores como, o acolhimento na instituição e o convívio com pessoas próximas. Faz-se importante agir para evitar estados de solidão e isolamento, muito comuns pelo afastamento da rede de apoio social até então conhecida (família, amigos, vizinhos, trabalho, grupos comunitários e religiosos, entre outros).

Sobre isso, a análise da relação entre fragilidade, sintomas depressivos e qualidade de vida de idosos institucionalizados, trazida pelo estudo descritivo de (Melo et al., 2018), em três ILPIs do interior do Estado de São Paulo, com 42 idosos (predominantemente do gênero feminino com média de idade de 77,4 anos), detectou 57,1\% sem sintomas depressivos e 38,1\% com sintomas característicos da depressão leve e $4,8 \%$ da depressão severa, e identificou que a fragilidade e os sintomas depressivos apresentaram correlação com a qualidade de vida dos idosos avaliados, nos domínios: Dignidade, Autonomia, Segurança e Individualidade Os impactos causados pela fragilidade contribuíram com a manifestação de sintomas depressivos, levando os acometidos a condição de fragilização.

Conforme (Frutuoso et al., 2019), com a transição demográfica no Brasil, o número de pessoas que passou a fazer parte da população de idosos aumentou de forma significativa e em paralelo, crescem gradativamente as perturbações de humor, típicas dessa fase da vida, inserindo-se os de ordem depressiva.

$\mathrm{Na}$ análise referente à prevalência de sintomas depressivos em 23 idosos com idade entre 60 e 89 anos, institucionalizados, realizada em três instituições de longa permanência (ILP), no município de Cajazeiras - PB, (Frutuoso et al., 2019), evidenciaram a prevalência de sintomas depressivos em $47,8 \%$ da sua amostra (39,1\% apresentaram nível leve e 8,7\% nível severo), sendo mais frequentes nos idosos com filhos, nos que não tinham uma boa autoavaliação de saúde, nos que não praticavam uma atividade física e no gênero feminino.

De acordo com (Fagundes et al., 2017), a prevalência de sintomas depressivos entre os idosos que residem em ILPI é 
mais elevada do que entre aqueles que moram com suas famílias, considerando que essa modalidade de atendimento e acolhimento pode não garantir o cuidado necessário ao residente. O sentimento de tristeza, ou de solidão, por exemplo, pode estar relacionado ao tratamento que the é direcionado, à perda da identidade e da individualidade, o que costuma ocorrer durante a institucionalização.

No estudo epidemiológico de (Guimaraes et al., 2019) para verificar a prevalência e fatores associados a sintomas depressivos em 42 idosos residentes em uma ILPI, foi encontrada a presença de sintomas depressivos em 54,8\%, predominando o sexo feminino $(64,7 \%)$, assim como verificaram a alta prevalência desses sintomas associados às variáveis presença de incontinência urinária, autopercepção de saúde, qualidade de sono e aposentadoria.

No estudo de (Maia et al., 2020), cujo objetivo consistiu em verificar a presença da depressão em 191 idosos institucionalizados, no universo de cinco abrigos da região da Mata Norte de Pernambuco (cidades de Timbaúba, Macaparana, Nazaré da Mata e Carpina), a fim de responder, dentro de um contexto local, se existe uma possível relação entre a depressão e a situação de exclusão e abandono em relação ao resto da sociedade nos quais se encontram tais idosos, observou-se que, dentro da população idosa institucionalizada tem- se uma alta prevalência de casos de depressão diagnosticada ou de suspeitas de depressão, acima da média da população geral brasileira e mundial.

\section{Conclusão}

Tal como o exposto, se reconhece que o aumento do envelhecimento populacional é uma realidade nacional e se trata de um processo que, conforme fatores como estilo de vida, condições socioeconômicas e doenças crônicas, podem variar de um sujeito para outro e impactar a sua qualidade de vida. Além dos fatores advindos do envelhecimento, os idosos podem ser afetados por sintomas de depressão, principalmente quando vivencia um processo de institucionalização.

Assim, se pode dizer, com base nas evidências das dez publicações analisadas, que houve a prevalência de sintomas depressivos nas ILPIs, campo de investigações das pesquisas. Esses sintomas depressivos, em seus níveis típicos, identificados na maior parte dos idosos institucionalizados, foram associados à diversas variáveis, como: a presença de comorbidades, incapacidade funcional, pouco engajamento nas atividades e reduzida participação social.

Contudo, verificou-se que a depressão é uma realidade no âmbito das ILPIs. A pesquisa constatou que essa, alcançou uma elevação preocupante entre idosos institucionalizados, levando a concluir que, dentre os desencadeadores da patologia, o tempo de institucionalização, a carência das relações interpessoais e a solidão constituíram em predominância, fatores de risco para o grau de depressão apresentada pelos sujeitos avaliados.

Desse modo, sugere-se que os dados dessa pesquisa contribuam para novas produções e investigações relacionadas ao quadro de envelhecimento no Brasil, e nesse contexto, o aumento de doenças cronicodegenerativas entre idosos institucionalizados.

\section{Referências}

Alves, M. B., et al,. (2017) Instituições de longa permanência para idosos: aspectos físico-estruturais e organizacionais. Esc. Anna Nery, 21(4), 01.

Belasco, A. G., \& Okuno, M. F. P. Realidade e desafios para o envelhecimento. REBEn - Rev. Bras. Enfermagem, 72,1 - 2.

Confortin, S. C. et al. (2017). Condições de vida e saúde de idosos: resultados do estudo de coorte EpiFloripa Idoso. Epidemiol. Serv. Saúde, $26,305-317$.

Damasceno, D. G., Chivelli, C. A., \& Lazinini, M. Q. (2019). Cuidando de idosos institucionalizados: representações de gestores e profissionais. Esc. Anna Nery, 23, 01 .

Fagundes, K. V. D. L. et al.. (2017). Instituições de longa permanência como alternativa no acolhimento das pessoas idosas. Rev. Salud Pública, 19, 210-214, 2017. 
Research, Society and Development, v. 10, n. 10, e130101018620, 2021 (CC BY 4.0) | ISSN 2525-3409 | DOI: http://dx.doi.org/10.33448/rsd-v10i10.18620

Frutuoso, E. A. et al. (2019). Idosos institucionalizados e depressão: rastreamento dos sintomas. Enfermagem Brasil, 18, $422-429$.

Gullich, I., Duro, S. M. S., \& Cesar, J. A. (2016). Depressão entre idosos: um estudo de base populacional no Sul do Brasil. Rev. Bras. Epidemiologia, 19, 691701 ,

Guimarães, L. A. et al.,(2019). Sintomas depressivos e fatores associados em idosos residentes em instituição de longa permanência. Ciênc. Saúde Coletiva, 24, $3275-3282$,

Hartmann Junior, J. A. S., \& Gomes, G. C. (2016). Depressão em idosos institucionalizados: padrões cognitivos e qualidade de vida. Ciências \& Cognição, 21, $137-154$.

Kratz, V. C. L. et al., (2018). Promoção de saúde de idosos institucionalizados e crenças quanto ao envelhecer: projeto intergeracional. Revista Saúde e Pesquisa, $11,277-286$.

Lampert, C. D. T., \& Ferreira, V. R. T. (2018). Fatores associados à sintomatologia depressiva em idosos. Aval. Psicol. Itatiba, 17,205 - 212.

Lopes, V. M. et al.,(2018). O que levou os idosos a institucionalização. Rev. Enferm UFPE, 12. 2428 - 2435.

Magalhães J. M. et al., (2016). Depressão em idosos na estratégia saúde da família: uma contribuição para a atenção primária. REME - Rev. Min Enfermagem, $20 \mathrm{e} 947, .01$.

Maia, R. P. et all. Depressão em idosos institucionalizados. Temas em Saúde, 20, 314 - 326.

Martins, E. F., \& Guimarães, F. P., (2017). Perfil dos idosos de uma instituição de longa permanência de uma cidade do interior de Minas Gerais. Rev. Brasileira de Ciências da Vida, 5, $01-22$.

Matias, A. G. C. et al.(2016). Indicadores de depressão em idosos e os diferentes métodos de rastreamento. Einstein, 14,6 - 11.

Melo, L. A. et al., (2018). Fragilidade, sintomas depressivos e qualidade de vida: um estudo com idosos institucionalizados. Rev baiana enfermagem, 32, 0109.

Miranda, G. M. D., Mendes, A. C. G., \& Silva, A. L. A. (2016). O envelhecimento populacional brasileiro: desafios e consequências sociais atuais e futuras Rev.bras. geriatr. Gerontol. 19, 507-519.

Moraes, B. S. et al., (2016). Sintomas da depressão associada ao abandono em idosos institucionalizados nos municípios de Firminópolis e São Luís De Montes Belos-Goiás. Revista Faculdade Montes Belos, 9, 106-141.

Nóbrega, I. P., Leal, M. C. C., \& Marques, A. P. O. (2016). Prevalência de sintomas depressivos e fatores associados em idosos institucionalizados no município de Recife, Pernambuco. Estud. Interdiscipl. Envelhec. 21, 135-154.

Pinheiro, N. C. G. et al., (2016). Desigualdade no perfil dos idosos institucionalizados na cidade de Natal, Brasil. Ciênc. Saúde coletiva, 21, 3399 - 3405.

Raldi, G. V., Cantele, A. B., \& Palmeiras, G. B. (2016). Avaliação da prevalência de depressão em idosos institucionalizados em uma ILPI no norte do RS. Revista de Enfermagem, 12, 48-63.

Silva, C. S. et al., (2016) A prevalência e alguns fatores de depressão em idosos institucionalizados. PUC-Goiás, 01 - 24.

Souza, M. T., Silva, M. D. \& Carvalho, R. (2010) Revisão integrativa: o que é e como fazer. Einstein, 8, 102 - 106.

Veras, R. P., \& Oliveira, M. (2018). Envelhecer no Brasil: a construção de um modelo de cuidado. Ciênc. saúde coletiva, 23,1929 - 1936. 